\title{
Genetic Variant of CXCR1 (rs2234671) Associates with Clinical Outcome in Perihilar Cholangiocarcinoma
}

\author{
Isabella Lurje ${ }^{a, b}$ Zoltan Czigany ${ }^{b}$ Jan Bednarsch ${ }^{b}$ Nadine Therese Gaisa ${ }^{c}$ \\ Edgar Dahlc $^{c}$ Ruth Knüchel ${ }^{c}$ Hannah Miller ${ }^{a, d}$ Tom Florian Ulmer ${ }^{b}$ \\ Pavel Strnad $^{\mathrm{e}}$ Christian Trautwein $^{\mathrm{e}}$ Frank Tacke $^{\mathrm{a}, \mathrm{e}}$ Ulf Peter Neumann ${ }^{\mathrm{b}, \mathrm{f}}$ \\ Georg Lurje b, d
}

aDepartment of Hepatology and Gastroenterology, Campus Charité Mitte, Campus Virchow-Klinikum,

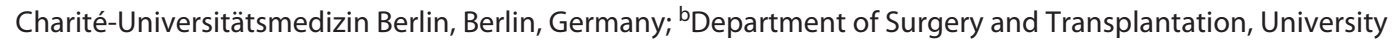
Hospital RWTH Aachen, Aachen, Germany; Institute of Pathology, University Hospital RWTH Aachen, Aachen,

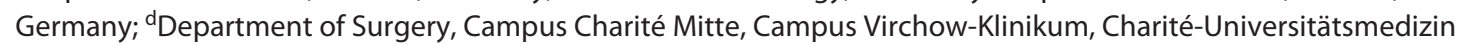
Berlin, Berlin, Germany; 'Department of Internal Medicine III, University Hospital RWTH Aachen, Aachen, Germany; fDepartment of Surgery, Maastricht University Medical Centre (MUMC), Maastricht, The Netherlands

\section{Keywords}

Perihilar cholangiocarcinoma Single-nucleotide polymorphism $\cdot$ IL-8 $\cdot$ CXCR1

\begin{abstract}
Background: Perihilar cholangiocarcinoma (pCCA) is a rare primary liver malignancy. Even in patients amenable to surgery, outcomes are often dismal. Here, we aimed to identify prognostic markers for patient outcomes by analyzing functionally relevant single-nucleotide polymorphisms (SNPs) in genes with a role in tumor inflammation and angiogenesis. We analyzed 11 polymorphisms in the inflammation-angiogenesis axis (VEGF, EGF, EGFR, IL-1b, IL-6, CXCL8 (IL-8), IL-10, $C X C R 1, H I F 1 A$, and $C O X 2$ genes) for their prediction of tumor recurrence and survival in PCCA patients undergoing surgery in a curative intent. Methods: Samples were obtained from 111 patients with pCCA undergoing liver resection in curative intent. DNA was extracted and analyzed using polymerase chain reaction-restriction fragment length polymorphism protocols and correlated with patients' outcomes. $\boldsymbol{R e}$ sults: Out of the assessed variants, only the CXCR1 (also: in-
\end{abstract}

Karger@karger.com www.karger.com/lic

Karger"

bOPEN ACCESS
(C) 2022 The Author(s).

Published by S. Karger AG, Basel

This is an Open Access article licensed under the Creative Commons Attribution-NonCommercial-4.0 International License (CC BY-NC) (http://www.karger.com/Services/OpenAccessLicense), applicable to the online version of the article only. Usage and distribution for commercial purposes requires written permission. terleukin-8-receptor alpha - IL-8RA) $+860 C>$ G heterozygous polymorphism (rs2234671) was associated with decreased disease-free survival (DFS), cancer-specific survival (CSS), and overall survival (OS) (18/111 (16.2\%), median DFS 14 months, log-rank $p=0.007$; median CSS 31 months, log-rank $p=0.007$; and median OS 6 months, $\log$-rank $p=0.002)$, compared to the GG genotype (92/111 (82.9\%), median DFS 55 months, median CSS 63 months, and median OS 33 months). In the multivariate analysis, $+860 \mathrm{C}>\mathrm{G}$ remained an independent prognostic factor for DFS (adjusted $p=0.008$ ), CSS (adjusted $p=0.001$ ), and OS (adjusted $p=0.001$ ). Conclusion: Genetic variant of $C X C R 1+860 C>G$ may serve as a molecular marker for DFS, CSS, and OS in patients undergoing curativeintent surgery for PCCA, indicating that the analysis of SNPs in genes involved in immune-mediated angiogenesis may help to identify patient subgroups at high risk for dismal oncological and overall outcome.

(C) 2022 The Author(s)

Published by S. Karger AG, Basel

I.L. and Z.C. and U.P.F. and G.L. contributed equally to this work as first and last authors, respectively.
Correspondence to:

Georg Lurje, georg.lurje@ charite.de 


\section{Introduction}

Cholangiocarcinoma (CCA) is the second most common primary liver cancer. A rising mortality from CCA has been reported worldwide during the last decade [1], despite the improvement of surgical and palliative treatment $[2,3]$. More than $50 \%$ of CCAs originate from the proximal extrahepatic bile ducts, are termed perihilar CCA (pCCA), and constitute the most common CCA entity in the Western world [4]. Many pCCA cases are sporadic, arising in the absence of the known risk factors such as chronic biliary inflammation, cholestasis, hepatobiliary parasitic infections, and liver cirrhosis [1]. Clinicopathological characteristics such as lymph node status and poor differentiation remain the best-studied, but imperfect prognostic, factors $[5,6]$. Therefore, the identification of preoperatively available molecular markers of prognosis as an adjunct to traditional staging systems may facilitate selection of patients who may benefit from surgical and adjuvant treatment strategies.

Angiogenesis - the induction of tumor neovascularization - is a prerequisite for tumor growth and survival. Over 50 years ago, Judah Folkman pioneered the hypothesis that vascular endothelial growth factor (VEGF) is a central driver of angiogenesis in solid malignancies, and since then, the predominant role of VEGF for tumor angiogenesis has become apparent [7]. More recently, it has been shown that the early angiogenic response to VEGF is also dependent on the interplay with immune cells that confer paracrine proliferative effects on the endothelium. As such, secreted VEGF attracts circulating monocytes and stimulates angiogenesis by forming SDF-1 traps [8]. In this regard, several cell populations in the tumor microenvironment (TME), such as myeloid-derived suppressor cells, tumor-associated macrophages, and cancer-associated fibroblasts (CAFs), support tumor growth, angiogenesis, and immune escape [9]. It has been proposed that the latter may play a crucial role in the regulation of inflammation and early-onset angiogenesis and in turn may impact the process of tumor growth and disease progression [10-12]. In addition, IL-8 has been reported to play a major role in VEGF-independent tumor angiogenesis in gastric and colon cancer $[13,14]$. In particular, an induction of IL-8 preserved the angiogenic phenotype in HIF1- $\alpha$-deficient colon cancer cells [15]. The G-protein-coupled receptor for IL-8, CXCR1, is expressed on granulocytes, monocytes, mast cells, and some natural killer cells [16] and is often overexpressed in malignant cells and their TME, where it confers proangiogenic and immunosuppressive effects $[17,18]$. We hypothesized that functional VEGF, EGF, EGFR, IL-1b, IL-6, IL-8, IL10, CXCR1 (IL-8RA), HIF1a, and COX2 polymorphisms could be associated with differences in clinical outcome in a large Western cohort of pCCA treated with curativeintent surgery.

\section{Materials and Methods}

\section{Study Population and Pathology}

A total of 113 unrelated consecutive patients with localized pCCA without signs of systemic disease underwent curative-intent surgery at the University Hospital RWTH Aachen between October 2010 and September 2019. Patients undergoing associating liver partition and portal vein ligation for staged hepatectomy $(n=2)$ were excluded from the analysis because associating liver partition and portal vein ligation for staged hepatectomy is not considered the mainstay of treatment in CCA, but rather a rescue operation in a small subset of patients with insufficient liver regeneration, where it is associated with dismal prognosis $[3,19]$. Clinicopathological and survival data were obtained from a prospectively managed institutional database and a senior hepatobiliary pathologist (NTG) reviewed the tumor histology to confirm appropriate patient selection. The Institutional Biobank (RWTH-cBMB) and the Department of Pathology provided patient material for genotyping (NTG, ED, and RKC). This study was conducted after approval by the Institutional Review Board of the RWTH Aachen University (EK 360/15 and EK 173/06) and in accordance with good clinical practice guidelines and the current Declaration of Helsinki.

\section{Staging and Surgical Technique}

All patients who were referred for surgical treatment of pCCA to our institution underwent a detailed clinical workup. None of the patients underwent neoadjuvant chemotherapy. Intrahepatic and distal CCA were not included in this analysis due to the markedly different tumor biology and surgical treatment. The preoperative workup included appropriate cross-sectional imaging to rule out the presence of distant metastases and CT or dynamic magnetic resonance imaging of the liver to visualize the invasion of major vessels in the liver hilum and an endoscopic retrograde cholangiopancreatography or magnetic resonance cholangiopancreatography to assess the extent of disease in the liver hilum. In selected cases, patients with suspected extrahepatic disease on conventional cross-sectional imaging underwent positron emission tomography-CT. Patients with extrahepatic disease were not included in this study. The surgical procedure for pCCA was carried out as previously described by Neuhaus et al. [20, 21]. Briefly, a "no-touch" hilar en bloc resection approach, as defined by extended liver resection with portal vein resection and reconstruction, was carried out in all cases $[2,6]$. Lymphadenectomy of the pericholedochal, periportal, common hepatic, posterior pancreaticoduodenal, and the celiac lymph nodes was routinely performed. All surgical specimens underwent routine histopathological workup according to current national guidelines, WHO- and UICC classifications. Tumor type, histopathological grading, and staging, loco-regional lymph node metastasis, resection margins, and vessel invasion were evaluated by an experienced board-certified staff pathologist. 
Table 1. Primer sequences, restriction enzymes, and reaction conditions

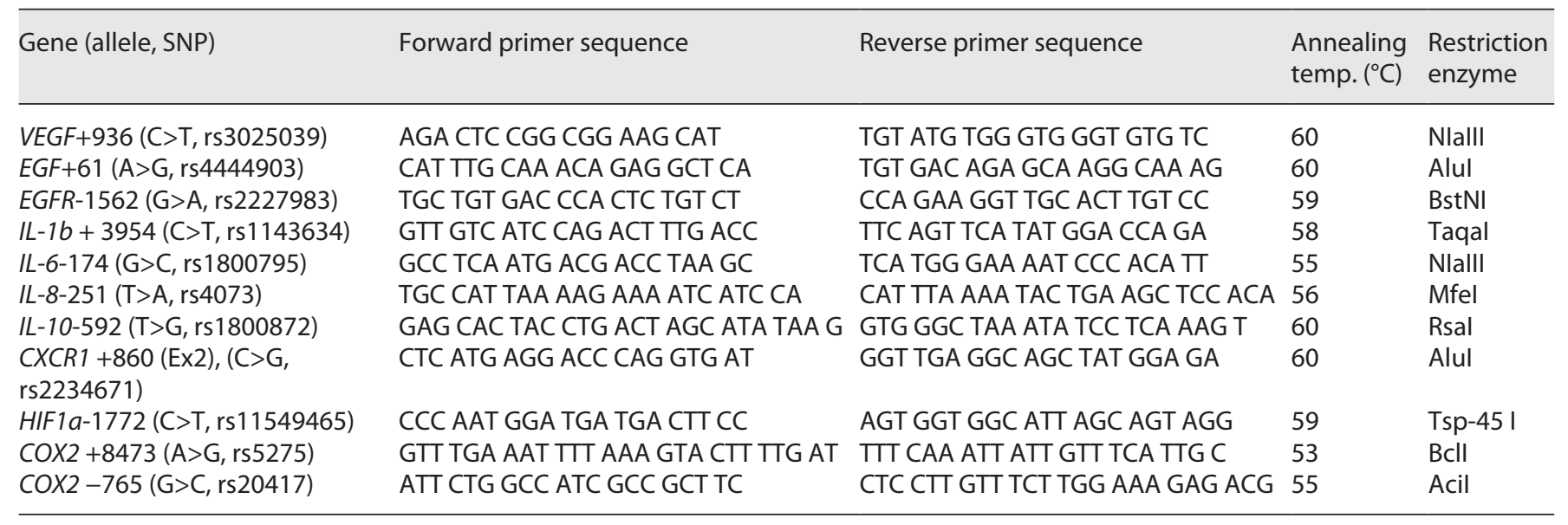

COX, cyclooxygenase; CXCR, chemokine receptor; EGF, epidermal growth factor; EGFR, epidermal growth factor receptor; HIFa, hypoxiainducing factor alpha; IL, interleukin; SNP, single-nucleotide polymorphism; VEGF, vascular endothelial growth factor.

\section{Single-Nucleotide Polymorphism Selection}

The polymorphisms we tested were selected by a pathway approach with the goal of selecting genes known to modulate inflammation and tumor-associated angiogenesis (Table 1). We used the following criteria to select genes for study: (a) that the gene is a part of a pathway for which there is evidence to support its involvement in tumor angiogenesis; (b) that the gene has a well-documented genetic polymorphism with an expected biological relevance; and (c) that the frequency of the polymorphism is high enough to enable a statistically meaningful association with clinical outcome. A total of 11 single-nucleotide polymorphisms (SNPs) in 10 genes were selected, including VEGF, EGF, EGFR, IL-1B, IL-6, CXCL8 (IL-8), IL-10, CXCR1 (IL-8RA), HIF1A, and COX2 (Table 1).

\section{Genotyping}

Formalin-fixed paraffin-embedded nontumor tissues were collected and genomic DNA was extracted using the QIAamp DNA extraction kit (Qiagen, CA, Valencia, USA) according to the manufacturer's protocol. Photometric analysis of DNA quality and content was conducted (NanoDrop, Thermo Fisher, Waltham, MA, USA). Polymerase chain reaction-restriction fragment length polymorphism technique was used for genotyping, as previously described $[22,23]$. Briefly, forward- and reverse primers flanking the SNP region were used for amplification and the amplicon was then digested with appropriate DNA restriction endonucleases (New England Biolabs, Ipswich, MA, USA) (Table 1). The reaction products were separated using agarose gel electrophoresis with a $4 \%$ agarose gel at $120 \mathrm{mV}$ for $60 \mathrm{~min}$ and visualized (GelDoc, BioRad Laboratories GmbH, Feldkirchen, Germany). For quality assurance purposes, a total of $10 \%$ positive and negative samples were randomly selected for each polymorphism and genotyped with genotype concordance $\geq 99 \%$.

\section{Endpoints and Statistical Analysis}

Patient outcome was assessed with the endpoints disease-free survival (DFS), defined as the period between surgery and first recurrence; cancer-specific survival (CSS), defined as the period be- tween surgery and death in those patients that recurred while patients with other causes of death were censored at the time of death; and overall survival (OS), defined as the period between liver resection and death without censoring for perioperative mortality. For DFS, patients were censored if they died without recurring, whereas patients lost to follow-up were censored at the time of last contact. Differences were tested using a two-tailed Fisher's exact test and a $\chi^{2}$ test for categorical variables and the Mann-Whitney $\mathrm{U}$ test for continuous variables as well as the Kruskal-Wallis test for nonparametric samples with more than 2 groups. Differences in DFS and OS between genotypes were assessed with Kaplan-Meier survival curves and the log-rank test. For SNPs with a frequency of the homozygous minor allele of $<10 \%$ in the study population, associations between genotypes and clinical outcome were analyzed in a dominant model. Otherwise, a codominant or additive model was employed. Associations of factors with DFS, CSS, and OS were analyzed with univariable and multivariable Cox proportional hazard models, with hazard ratios presented with $95 \%$ confidence intervals (CI). Significant variables in the univariable analyses were included in the multivariable analyses. The level of significance was set to $p<0.05$. Analyses were performed with SPSS Statistics (v23, IBM Corp., Armonk, NY, USA).

\section{Results}

A total of 111 patients with localized pCCA were included in this study. The cohort comprised 75 (68\%) men and $38(32 \%)$ women with a median age of 66 years at the time of surgery. Six $(6 / 111,5 \%)$ of pCCAs were classified as Bismuth I, 8 (7\%) as Bismuth II, 30 as Bismuth IIIa (27\%), 32 as Bismuth IIIb (29\%), and 35 as Bismuth IV (32\%). Thirty-six patients had preoperative cholangitis $(36 / 111,32 \%)$. Due to cholestasis, 88 (80\%) patients re- 


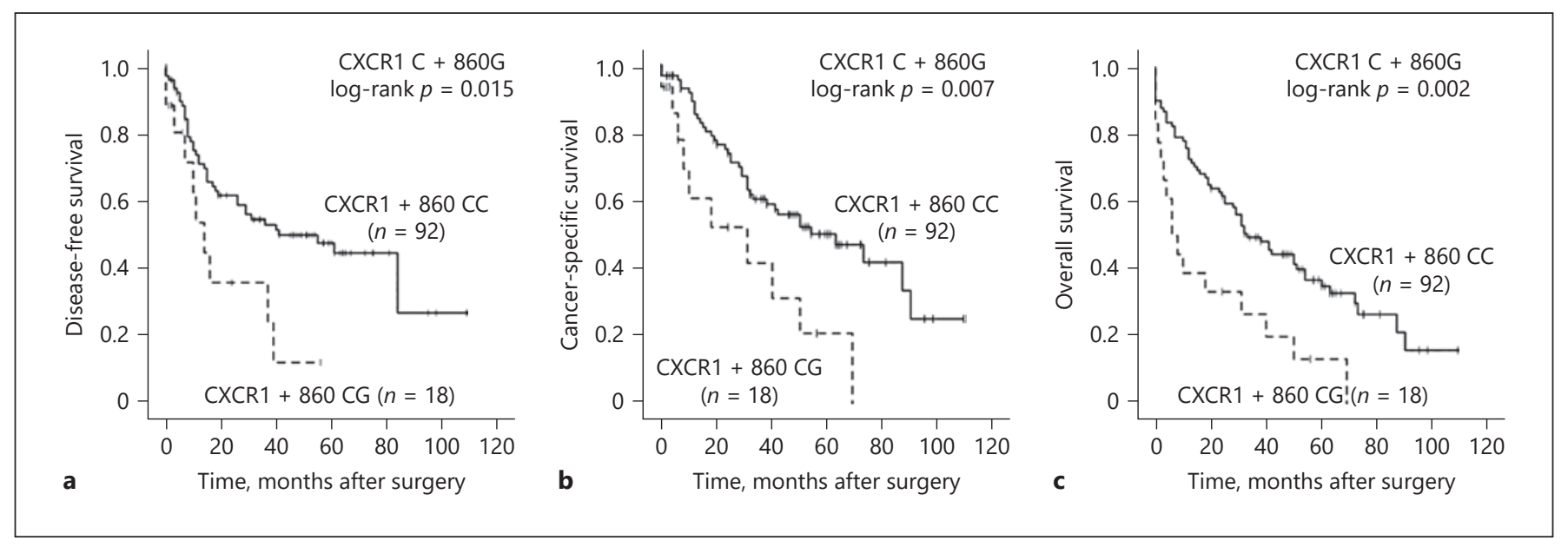

Fig. 1. DFS (a), CSS (b), and OS of patients (c) by CXCR1 polymorphism.

ceived preoperative endoscopic biliary drainage and/or percutaneous biliary drainage $(26 / 111,23 \%)$. Portal vein embolization was performed in 47 (42\%) patients. No patient received neoadjuvant chemotherapy. All patients received systematic lymphadenectomy $(111 / 111,100 \%)$ and most patients $(103 / 111,93 \%)$ underwent vascular resection with reconstruction. One patient $(1 / 111,1 \%)$ received an anatomical resection, 9 (8\%) and 13 (12\%) underwent right and left hepatectomy, respectively, while 20 $(18 \%)$ and $30(27 \%)$ underwent extended right and left hepatectomy, respectively. Twenty-three (23/111, 21\%) patients were treated with right trisectionectomy, 6 patients (5\%) with left trisectionectomy, and 9 patients (8\%) with hepatoduodenectomy (extended patient data in online suppl. Table 1; for all online suppl. material, see www. karger.com/doi/10.1159/000521613). Median follow-up was 32 months. DFS was 37 months, and 51 patients $(46 \%)$ recurred in the observation time. Median CSS was 50 months, with 50 patients $(45 \%)$ recurring prior to death. Median OS was 31 months, with 78 patients (70\%) deceased during the observation period.

\section{CXCR1 G+860C and Outcome Parameter}

Of the 11 tested polymorphisms, only CXCR $1 \mathrm{G}+860 \mathrm{C}$ correlated significantly with outcome parameters. Genotyping for CXCR $1 \mathrm{G}+860 \mathrm{C}$ was successful in $99.0 \%$ of cases $(110 / 111)$. Ninety-two patients $(83 \%, 92 / 111)$ of were homozygous for the CXCR $1+860$ C allele, 16\% (18/111) heterozygous $(\mathrm{C} / \mathrm{G})$, and no patient was homozygous for the $C X C R 1+860 \mathrm{G}$ allele. When analyzing the association of the CXCR1 +860 polymorphism with postoperative complications, patients with the $\mathrm{C} / \mathrm{G}$ genotype had a sig- nificantly higher rate of postoperative 90-day mortality $(6 / 18,33.3 \%)$ than patients with the wild-type alleles $(12 / 92,13.0 \%, p=0.37)$. No significant difference regarding 90-day Clavien-Dindo $\geq$ CD3b complications, intensive care, and hospital stay, and 90-day comprehensive complication index (CCI) was noted (online suppl. Table 2 ). The G+860C genotype was equally distributed between Bismuth stages (online suppl. Table 3, $p=0.828$ ).

The CXCR $1+860$ polymorphism was significantly associated with all three outcome parameters. Patients homozygous for the CXCR1 $+860 \mathrm{C}$ allele (wild type) had a median DFS of 55 months (95\% CI: 21.6-88.4), while patients with the G/C genotype had a median DFS of 14 months (95\% CI: 7.7-20.3, log-rank $p=0.015$, Fig. 1a). Forty-five percentage (41/92) of patients homozygous for the $\mathrm{C}$ allele and 55\% (10/18) of heterozygous patients $(\mathrm{C} / \mathrm{G})$ recurred during the observation time. The CXCR1 +860 polymorphism showed a significant association with CSS. Patients with the $\mathrm{C} / \mathrm{C}$ homozygous genotype had a median CSS of 63 months (95\% CI: 35.3-90.7), while patients with a C/G genotype had a median CSS of 31 months (95\% CI: 0.0-62.8, log-rank $p=0.007$, Fig. 1b). Forty-three percentage (40/92) of patients homozygous for the CXCR $1+860$ C allele and 55\% (10/18) of patients with a heterozygous $(\mathrm{C} / \mathrm{G})$ genotype died from their oncological disease. Median OS for patients homozygous for the CXCR1 +860C allele was 33 months (95\% CI: 21.7-44.3) and for patients with the CXCR $1+860 \mathrm{C} / \mathrm{G}$ genotype 6 months (95\% CI: $0.5-11.5, \log$-rank $p=0.002$, Fig. 1c). Sixty-six percentage (61/92) of patients with the CC genotype and $89 \%(16 / 18)$ of patients with the GC genotype deceased during the observation period (Table 2 ). 


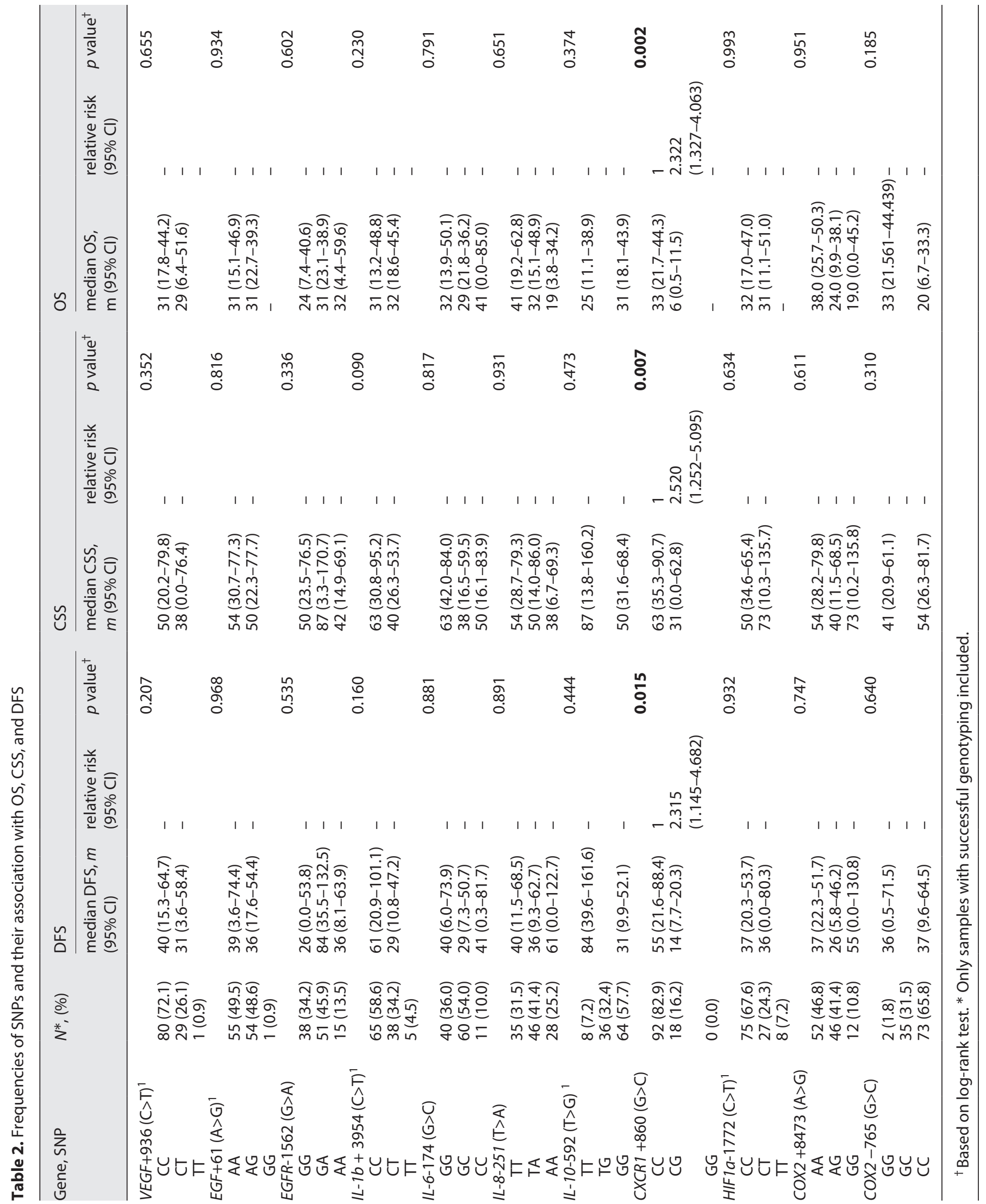




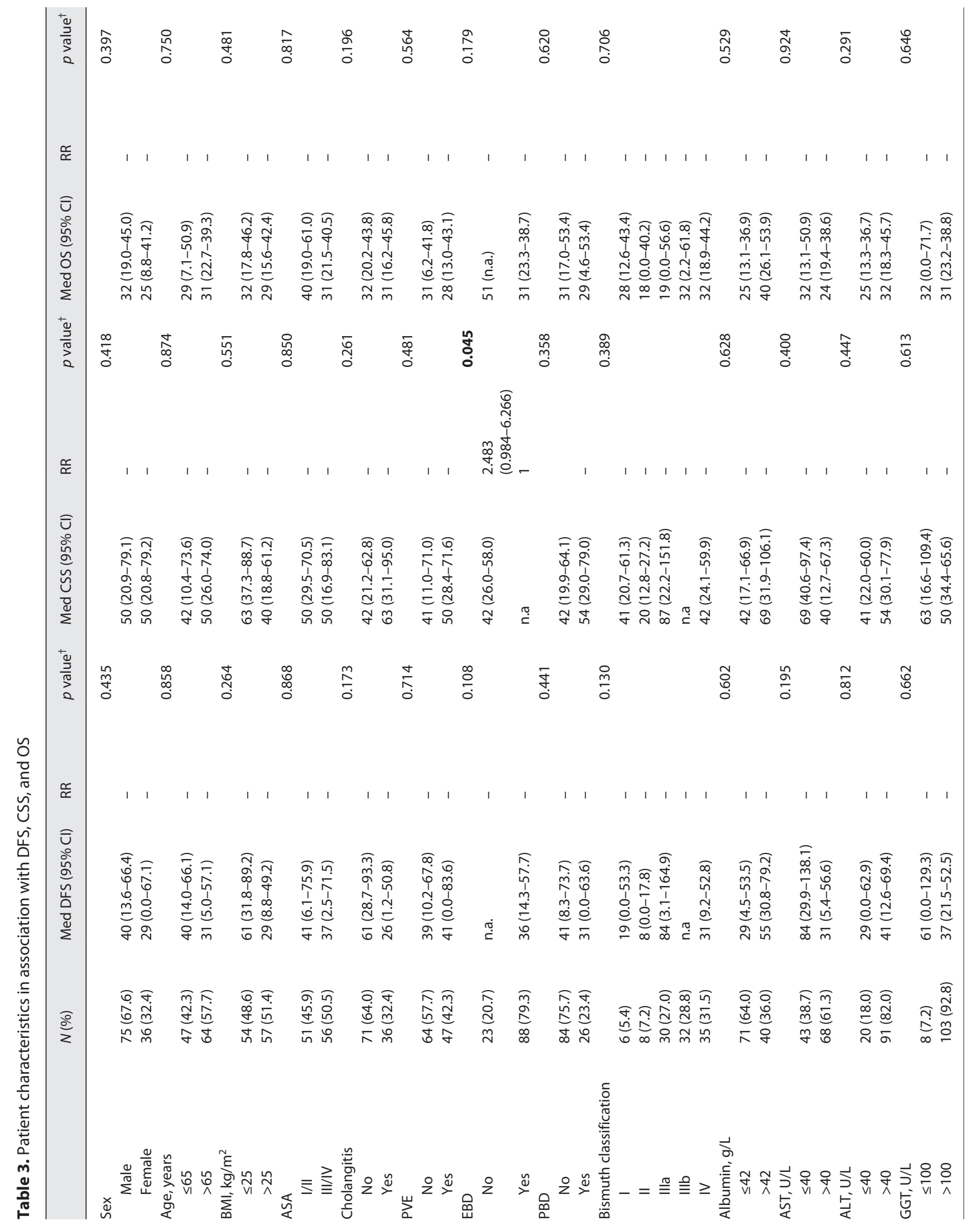




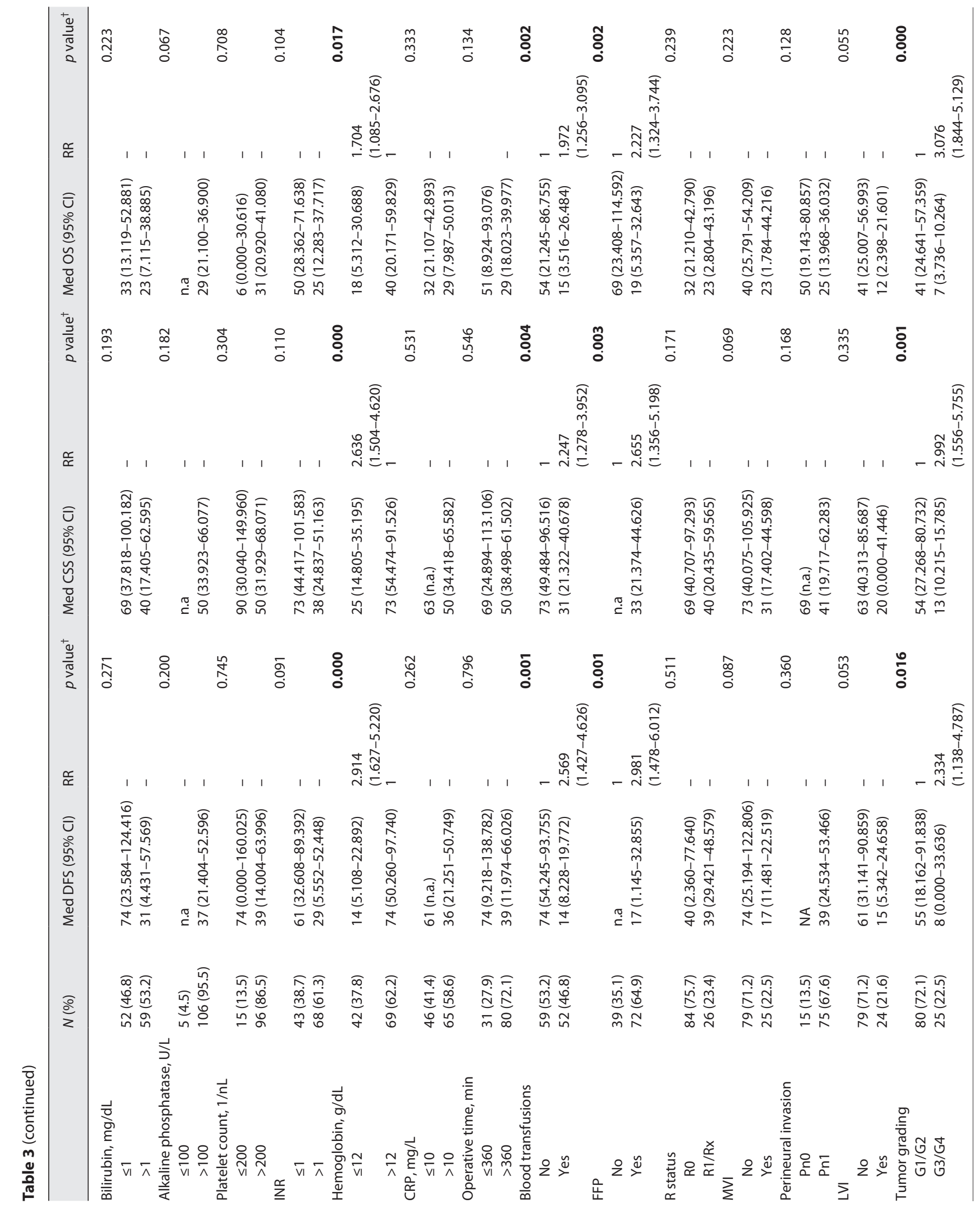




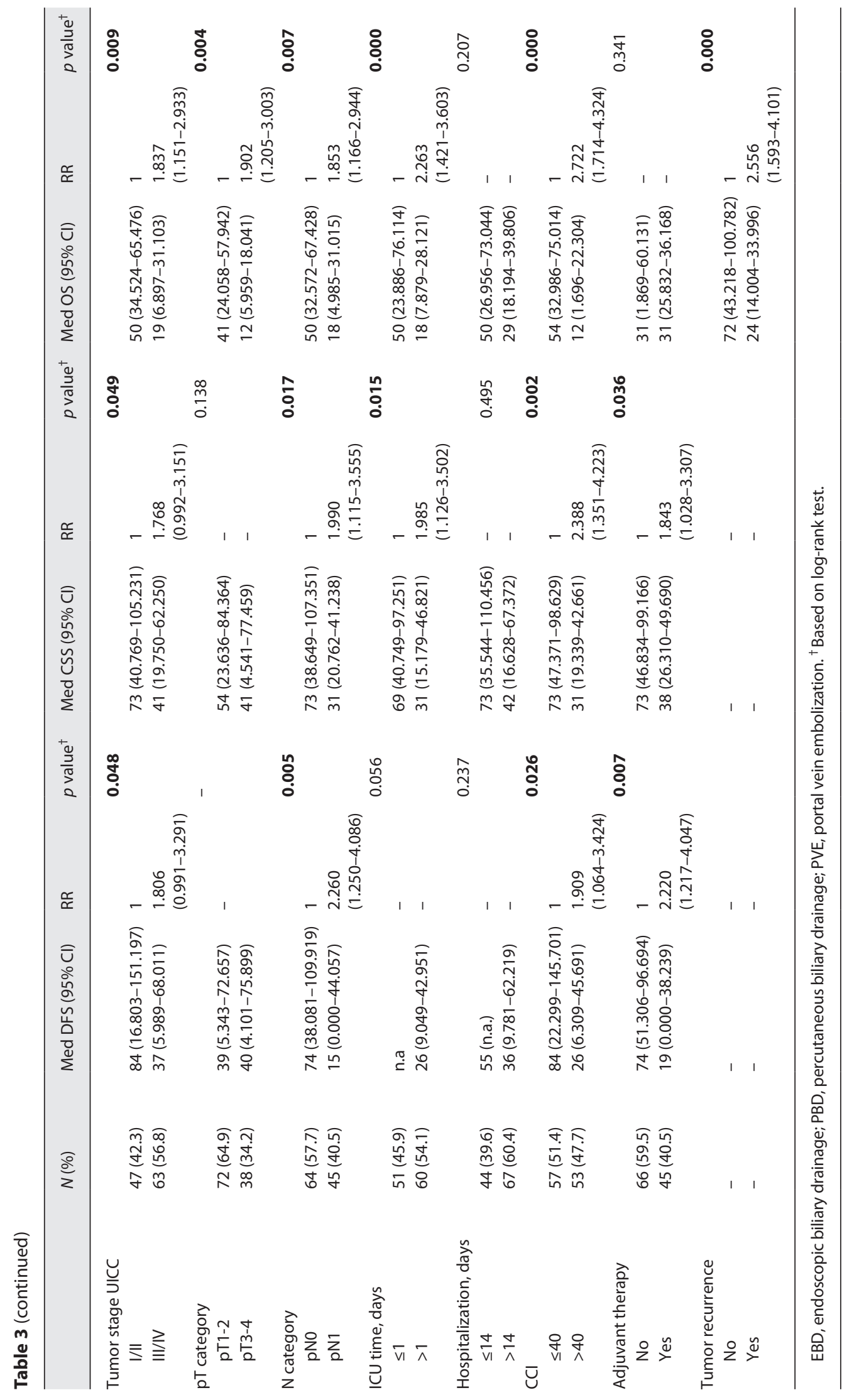




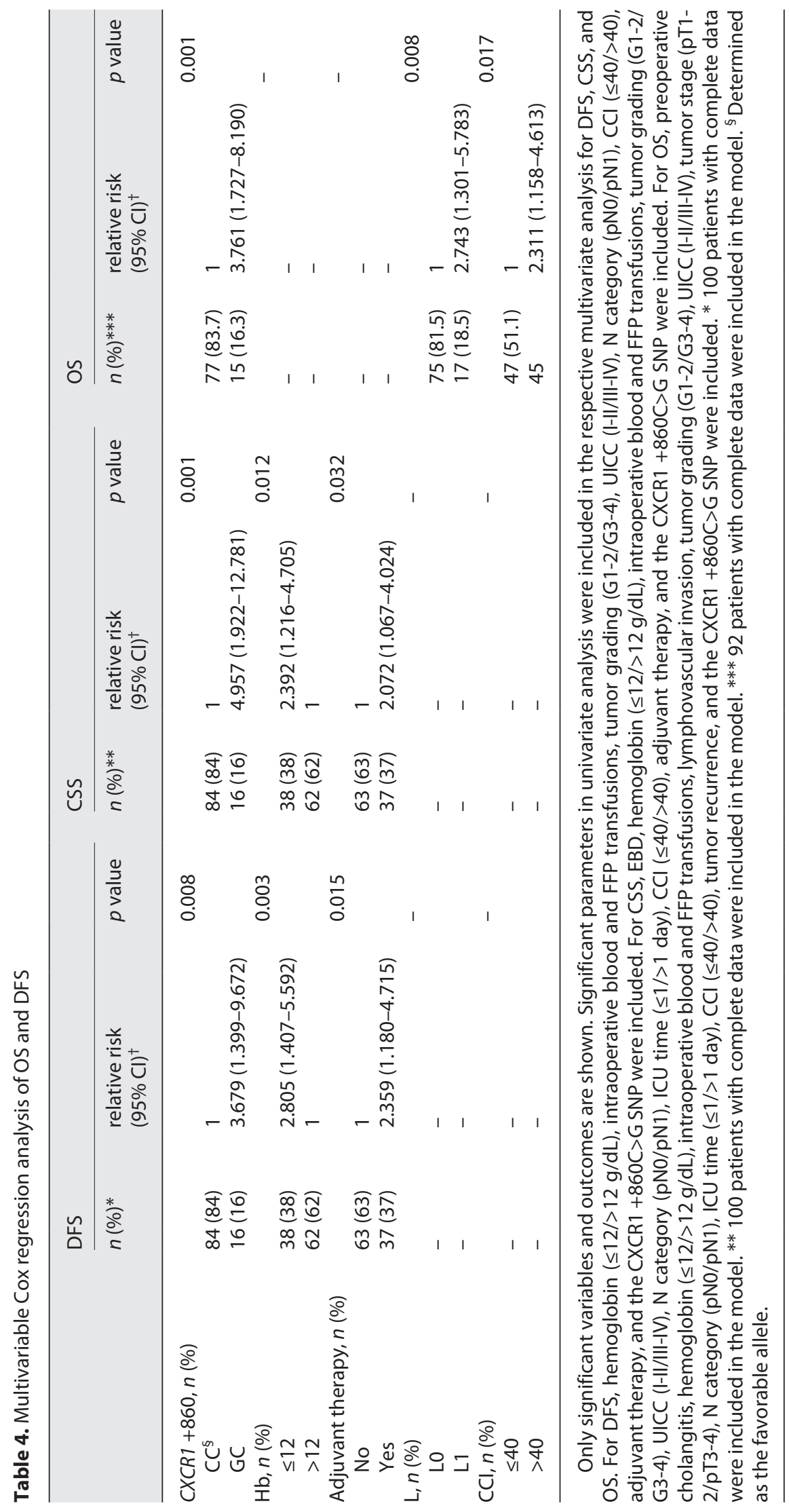


In the multivariable analysis incorporating significant clinicopathological parameters from the univariable analysis (Table 3), the CXCR1 rs2234671 polymorphism remained significantly associated with DFS, CSS, and OS. As such, the CXCR $1+860$ genotype (G/C relative risk [RR] 3.679 [95\% CI: 1.399-9.672] $p=0.008)$, preoperative hemoglobin ( $\leq 12$ RR 2.805 [95\% CI: 1.407-5.592] $p=$ 0.003 ), and adjuvant therapy (RR 2.359 [95\% CI: 1.1804.715] $p=0.015)$ were significantly associated with DFS. CXCR1 +860 (G/C RR 4.957 [95\% CI: 1.922-12.781] $p=$ 0.001), preoperative hemoglobin ( $\leq 12$ RR 2.392 [95\% CI $1.216-4.705] p=0.012$ ), and adjuvant therapy (RR 2.072 [95\% CI: 1.067-4.024] $p=0.032$ ) were also significantly associated with CSS. CXCR1 +860 (G/C RR 3.761 [95\% CI: 1.727-8.190] $p=0.001)$, lymph node status (L1 RR 2.743 [95\% CI: $1.301-5.783] p=0.008)$, and CCI ( $>40 \mathrm{RR}$ 2.311 [95\% CI: 1.158-4.613] $p=0.017$ ) were significantly associated with OS (Table 4).

\section{Discussion}

We were able to demonstrate that a germline polymorphism of CXCR1 independently predicts tumor recurrence and survival in a large homogenous patient group with pCCA. To the best of our knowledge, this is the first study to show that the $C X C R 1+860 \mathrm{C}>\mathrm{G}$ polymorphism may be an important prognostic marker for pCCA tumor relapse and overall survival, independent of lymph node status, hemoglobin, and perioperative complications.

pCCA is a rare but highly aggressive primary hepatic malignancy. While the last years have witnessed considerable advances in characterizing the genetic and transcriptomic landscape of CCA, the tumor biology of CCA remains understudied compared to other gastrointestinal malignancies [24]. Suggestions to expand traditional TNM staging with clinical adjuncts have been made [25], but relatively few biomarker-based staging systems were implemented so far $[26,27]$. The role of tumor neovascularization in cancer, especially the prominent role of VEGF, is well established [13, 28]. Tumor cells and immune cells from the TME, such as infiltrating CD14 (+) CD16 (+) monocytes and CAFs, abundantly secrete VEGF and promote angiogenesis and metastasis $[29,30]$. However, clinical studies investigating the efficacy of VEGF inhibition for CCA have reported overall discouraging results [31,32], suggesting that alternative mechanisms may support CCA neoangiogenesis. Besides directly stimulating neovascularization by secreting soluble angiogenic factors, malignant cells actively interact with the

Molecular Determinants of pCCA

Recurrence
TME to enhance neovascularization. In this regard, the inflammatory TME amplifies VEGF-dependent angiogenesis and promotes tumor progression through VEGFindependent pathways [8]. In turn, endothelial cells can attract leucocytes to facilitate angiogenesis, tumor growth, and metastasis. As such, the release of IL- 8 from endothelial cells recruits and activates granulocytes and monocytes and triggers their transformation into immunosuppressive myeloid-derived suppressor cells [17].

A prominent stromal reaction is inherent to pCCA, with a characteristic hypoxic microenvironment and a reduced vascularity in comparison to HCC [1]. A dense network of CAFs and endothelial cells surrounds the tumor and intensely interacts with the malignant cells as well as with resident and migratory immune cells [30]. In vitro studies have identified CAFs as a source of soluble mediators with direct proinvasive effects on cocultured CCA cells, with IL-8 as one of the main secreted mediators [11]. As such, excessive IL- 8 secretion by senescent CAFs drives invasion and metastasis in other cancer entities with a prominent desmoplastic reaction, such as pancreatic cancer $[14,33,34]$. Recent CCA single-cell sequencing data showed that the majority of CAFs in CCA exhibit a microvasculature signature with an upregulation of genes responsible for hypoxia response and are localized in proximity to microvascular regions, underlining their mediator role between malignant cells and neoangiogenesis. In vitro and in vivo data showed that the presence of vascular CAFs promotes CCA tumor growth [35]. While genetic variants of IL-8 and its receptor CXCR1 (also interleukin-8-receptor alpha-IL-8RA) were linked with clinical outcome in gastric and colon cancer $[13,14]$, the induction of IL-8 signaling preserved the angiogenic phenotype in HIF1- $\alpha$-deficient colon cancer cells, suggesting a critical role of IL-8 in tumor-associated angiogenesis, independent of VEGF [15]. These findings support our hypothesis that altered IL-8 signaling may impact immunosuppressive and proangiogenic signals in the TME.

The CXCR1 rs2234671 860C > G polymorphism causes a missense variant with a Ser $>$ Thr substitution at position 276 , located in the extracellular domain of the receptor $[36,37]$. While the polymorphism is not located in an expression quantitative trait locus, it seems likely that the polymorphism causes altered receptor signaling [38]. As such, the exchanged amino acid directly adjoins to the amino acid in position 277, which forms a disulfide bond to a cysteine side chain in position 30 [39], therefore potentially altering the tertiary protein structure of the receptor. 
The wild-type CXCR1 +860 alleles (CC) have been linked to improved response to bevacizumab (antiVEGF) and increased DFS in colorectal, pancreatic, lung, renal, breast, and gastric cancer [38], underlining the impact of the CXCR1 +860 SNP on VEGF-dependent angiogenesis. Previously, the CXCR1 rs2234671 polymorphism has been associated with an increased susceptibility to breast cancer [40]. To the best of our knowledge, the prognostic role of the $\mathrm{C}>\mathrm{G}$ polymorphism has not yet been investigated in CCA. Interestingly, patients with gallstones and carriers of the $C X C R 1+860 \mathrm{C}>\mathrm{G}$ polymorphism had a 26-fold risk for the development of gallbladder cancer, compared to those without gallstones [41]. Furthermore, high IL-8 protein expression in surgical pCCA cases has been associated with microvascular density and dismal survival [42].

When analyzing the genotype of the CXCR1 receptor polymorphism (rs2234671), we observed that carriers of the G-allele had inferior DFS, CSS, and OS in both univariable and multivariable analyses. The results of the present study must be interpreted with caution due to its retrospective nature. The findings from this study will need to be replicated in other studies on pCCA and validated in independent trials. Despite originating from a single-center cohort, a strength of this report is the rigorous definition of a homogenous patient collective with only treatment-naïve, perihilar disease, managed with a universal surgical approach of hilar en bloc resection with lymphadenectomy. Due to the relatively low incidence of pCCA, many single-center studies are conducted in cohorts with heterogeneous CCA localization and pretreatments, limiting the comparability of results [43].

Taken together and consistent with our hypothesis, a genetic variant of an immunoregulatory gene involved in tumor inflammation and angiogenesis independently predicted clinical outcome in patients undergoing curative-intent surgery for pCCA. Our findings suggest that the assessment of the patients' angiogenic potential based on CXCR1 genotypes may predict postoperative disease progression and survival. Potentially, this may aid the identification of novel therapeutic targets. Biomarkerembedded clinical trials and validation in an independent cohort of patients are required to confirm our findings.

\section{Statement of Ethics}

This research complies with the guidelines for human studies and was conducted ethically in accordance with the World Medical Association Declaration of Helsinki. This study protocol was reviewed and approved by the Institutional Review Board of the
RWTH Aachen University (EK 360/15 and EK 173/06). Written informed consent was not required due to the retrospective nature of the study (Institutional Review Board of the RWTH Aachen University, EK 360/15, EK 173/06).

\section{Conflict of Interest Statement}

The authors have no conflicts of interest to declare.

\section{Funding Sources}

This research project was in part supported by the START program (\#136/17 and \#23/19) of the Faculty of Medicine, RWTH Aachen, and the Excellence Initiative of the German federal and state governments (G:(DE-82) ZUK2-SF-OPSF443) without involvement of the funders in study design, data collection, data analysis, manuscript preparation, or decision to publish.

\section{Author Contributions}

The study was designed by the initiating study team (I.L., Z.C., G.L., U.P.N., and J.B.). Data collection and analysis were performed by I.L., Z.C., J.B., H.M., N.T.G., U.P.N., and G.L. Laboratory experiments were conducted by I.L. and H.K. The manuscript was drafted by I.L., Z.C., and G.L. Further authors (F.T., P.S., C.T., E.D., N.T.G., T.F.U., R.K., and U.P.N.) have substantially contributed to the final version of the manuscript. All authors have read and approved the final version of the manuscript.

\section{Data Availability Statement}

All relevant data were reported within the article. Further supporting data will be provided upon written request addressed to the corresponding author.

\section{References}

1 Banales JM, Marin JJG, Lamarca A, Rodrigues PM, Khan SA, Roberts LR, et al. Cholangiocarcinoma 2020: the next horizon in mechanisms and management. Nat Rev Gastroenterol Hepatol. 2020;17(9):557-88.

2 Bednarsch J, Czigany Z, Lurje I, Tacke F, Strnad P, Ulmer TF, et al. Left- versus rightsided hepatectomy with hilar en-bloc resection in perihilar cholangiocarcinoma. HPB. 2020;22(3):437-44.

3 Li J, Moustafa M, Linecker M, Lurje G, Capobianco I, Baumgart J, et al. ALPPS for locally advanced intrahepatic cholangiocarcinoma: did aggressive surgery lead to the oncological benefit? An international multi-center study. Ann Surg Oncol. 2020;27(5):1372-84.

4 DeOliveira ML, Cunningham SC, Cameron JL, Kamangar F, Winter JM, Lillemoe KD, et al. Cholangiocarcinoma: thirty-one-year experience with 564 patients at a single institution. Ann Surg. 2007;245(5):755-62. 
5 Mavros MN, Economopoulos KP, Alexiou VG, Pawlik TM. Treatment and prognosis for patients with intrahepatic cholangiocarcinoma: systematic review and meta-analysis. JAMA Surg. 2014;149(6):565-74.

6 Lurje G, Bednarsch J, Czigany Z, Lurje I, Schlebusch IK, Boecker J, et al. The prognostic role of lymphovascular invasion and lymph node metastasis in perihilar and intrahepatic cholangiocarcinoma. Eur J Surg Oncol. 2019;45(8):1468-78.

7 Bergers G, Benjamin LE. Tumorigenesis and the angiogenic switch. Nat Rev Cancer. 2003; 3(6):401-10.

8 Grunewald M, Avraham I, Dor Y, BacharLustig E, Itin A, Jung S, et al. VEGF-induced adult neovascularization: recruitment, retention, and role of accessory cells. Cell. 2006; 124(1):175-89.

9 Lurje I, Hammerich L, Tacke F. Dendritic cell and $\mathrm{T}$ cell crosstalk in liver fibrogenesis and hepatocarcinogenesis: implications for prevention and therapy of liver cancer. Int J Mol Sci. 2020;21(19):7378.

10 Ping Q, Yan R, Cheng X, Wang W, Zhong Y, Hou Z, et al. Cancer-associated fibroblasts: overview, progress, challenges, and directions. Cancer Gene Ther. 2021;28(9):984-99.

11 Heits N, Heinze T, Bernsmeier A, Kerber J, Hauser C, Becker T, et al. Influence of mTORinhibitors and mycophenolic acid on human cholangiocellular carcinoma and cancer associated fibroblasts. BMC Cancer. 2016;16:322.

12 Ning Y, Manegold PC, Hong YK, Zhang W, Pohl A, Lurje G, et al. Interleukin-8 is associated with proliferation, migration, angiogenesis and chemosensitivity in vitro and in vivo in colon cancer cell line models. Int J Cancer. 2011;128(9):2038-49.

13 Lurje G, Zhang W, Schultheis AM, Yang D, Groshen S, Hendifar AE, et al. Polymorphisms in VEGF and IL-8 predict tumor recurrence in stage III colon cancer. Ann Oncol. 2008;19(10):1734-41.

14 Lurje G, Husain H, Power DG, Yang D, Groshen S, Pohl A, et al. Genetic variations in angiogenesis pathway genes associated with clinical outcome in localized gastric adenocarcinoma. Ann Oncol. 2010;21(1):78-86.

15 Strieter RM. Masters of angiogenesis. Nat Med. 2005;11(9):925-7.

16 Chuntharapai A, Lee J, Hébert CA, Kim KJ. Monoclonal antibodies detect different distribution patterns of IL- 8 receptor A and IL-8 receptor B on human peripheral blood leukocytes. J Immunol. 1994;153(12):5682-8.

17 Alfaro C, Teijeira A, Oñate C, Pérez G, Sanmamed MF, Andueza MP, et al. Tumor-produced interleukin-8 attracts human myeloidderived suppressor cells and elicits extrusion of neutrophil extracellular traps (NETs). Clin Cancer Res. 2016;22(15):3924-36.

18 Liu Q, Li A, Tian Y, Wu JD, Liu Y, Li T, et al The CXCL8-CXCR1/2 pathways in cancer. Cytokine Growth Factor Rev. 2016;31:61-71.

19 Olthof PB, Coelen RJS, Wiggers JK, Groot Koerkamp B, Malago M, Hernandez-Alejan- dro R, et al. High mortality after ALPPS for perihilar cholangiocarcinoma: case-control analysis including the first series from the international ALPPS registry. HPB. 2017;19(5): 381-7.

20 Neuhaus P, Thelen A, Jonas S, Puhl G, Denecke T, Veltzke-Schlieker W, et al. Oncological superiority of hilar en bloc resection for the treatment of hilar cholangiocarcinoma. Ann Surg Oncol. 2012;19(5):1602-8.

21 Neuhaus P, Jonas S, Bechstein WO, Lohmann $\mathrm{R}$, Radke C, Kling N, et al. Extended resections for hilar cholangiocarcinoma. Ann Surg. 1999;230(6):808-18. discussion 819.

22 Lurje G, Nagashima F, Zhang W, Yang D, Chang HM, Gordon MA, et al. Polymorphisms in cyclooxygenase-2 and epidermal growth factor receptor are associated with progression-free survival independent of $\mathrm{K}$ ras in metastatic colorectal cancer patients treated with single-agent cetuximab. Clin Cancer Res. 2008;14(23):7884-95.

23 Rhodes KE, Zhang W, Yang D, Press OA, Gordon M, Vallböhmer D, et al. ABCB1, SLCO1B1 and UGT1A1 gene polymorphisms are associated with toxicity in metastatic colorectal cancer patients treated with firstline irinotecan. Drug Metab Lett. 2007;1(1): 23-30.

24 Li H, Qu L, Zhang H, Liu J, Zhang X. A comprehensive transcriptomic landscape of cholangiocarcinoma based on bioinformatics analysis from large cohort of patients. Sci Rep. 2021;11(1):13713.

25 Chaiteerakij R, Harmsen WS, Marrero CR, Aboelsoud MM, Ndzengue A, Kaiya J, et al. A new clinically based staging system for perihilar cholangiocarcinoma. Am J Gastroenterol. 2014;109(12):1881-90.

26 Macias RIR, Kornek M, Rodrigues PM, Paiva NA, Castro RE, Urban S, et al. Diagnostic and prognostic biomarkers in cholangiocarcinoma. Liver Int. 2019;39 Suppl 1:108-22.

27 Loosen SH, Lurje G, Wiltberger G, Vucur M, Koch A, Kather JN, et al. Serum levels of miR29, miR-122, miR-155 and miR-192 are elevated in patients with cholangiocarcinoma. PLoS One. 2019;14(1):e0210944.

28 Lurje G, Lenz HJ. EGFR signaling and drug discovery. Oncology. 2009;77(6):400-10.

29 Subimerb C, Pinlaor S, Lulitanond V, Khuntikeo N, Okada S, McGrath MS, et al. Circulating CD14(+) CD16(+) monocyte levels predict tissue invasive character of cholangiocarcinoma. Clin Exp Immunol. 2010;161(3):471-9.

30 Cadamuro $M$, Nardo $G$, Indraccolo $S$, Dall'olmo L, Sambado L, Moserle L, et al. Platelet-derived growth factor-D and Rho GTPases regulate recruitment of cancer-associated fibroblasts in cholangiocarcinoma. Hepatology. 2013;58(3):1042-53.

31 Shroff RT, Yarchoan M, O'Connor A, Gallagher D, Zahurak ML, Rosner G, et al. The oral VEGF receptor tyrosine kinase inhibitor pazopanib in combination with the MEK inhibitor trametinib in advanced cholangiocarcinoma. Br J Cancer. 2017;116(11):1402-7.
32 Lubner SJ, Mahoney MR, Kolesar JL, Loconte NK, Kim GP, Pitot HC, et al. Report of a multicenter phase II trial testing a combination of biweekly bevacizumab and daily erlotinib in patients with unresectable biliary cancer: a phase II Consortium study. J Clin Oncol. 2010;28(21):3491-7.

33 Wang T, Notta F, Navab R, Joseph J, Ibrahimov E, Xu J, et al. Senescent carcinoma-associated fibroblasts upregulate IL8 to enhance prometastatic phenotypes. Mol Cancer Res. 2017;15(1):3-14.

34 Lurje G, Leers JM, Pohl A, Oezcelik A, Zhang W, Ayazi S, et al. Genetic variations in angiogenesis pathway genes predict tumor recurrence in localized adenocarcinoma of the esophagus. Ann Surg. 2010;251(5): 857-64.

35 Zhang M, Yang H, Wan L, Wang Z, Wang H, Ge C, et al. Single-cell transcriptomic architecture and intercellular crosstalk of human intrahepatic cholangiocarcinoma. J Hepatol. 2020;73(5):1118-30.

36 Information NCfB. Reference SNP (rs) Report rs2234671: NIH National Library of Medicine; 2020 [updated 2021 Jun 8; cited 2020 Apr 21]. Available from: https://www. ncbi.nlm.nih.gov/snp/rs2234671\#frequency_ tab.

37 Liu Y, Yang S, Lin AA, Cavalli-Sforza LL, Su B. Molecular evolution of CXCR1, a G protein-coupled receptor involved in signal transduction of neutrophils. J Mol Evol. 2005; 61(5):691-6.

38 de Haas S, Delmar P, Bansal AT, Moisse M, Miles DW, Leighl N, et al. Genetic variability of VEGF pathway genes in six randomized phase III trials assessing the addition of bevacizumab to standard therapy. Angiogenesis. 2014;17(4):909-20.

39 Park SH, Das BB, Casagrande F, Tian Y, Nothnagel HJ, Chu M, et al. Structure of the chemokine receptor CXCR1 in phospholipid bilayers. Nature. 2012;491(7426):779-83.

40 Gallegos-Arreola MP, Briseño-Zuno CJ, Figuera LE, Sánchez-López JY, ZúñigaGonzález GM, Puebla-Pérez AM, et al. The rs1008562, rs2234671, and rs3138060 polymorphisms of the CXCR1 gene are associated with breast cancer risk in a Mexican population. Eur Rev Med Pharmacol Sci. 2020; 24(19):9990-10002.

41 Hsing AW, Sakoda LC, Rashid A, Andreotti G, Chen J, Wang BS, et al. Variants in inflammation genes and the risk of biliary tract cancers and stones: a population-based study in China. Cancer Res. 2008;68(15):6442-52.

42 Sun Q, Li F, Sun F, Niu J. Interleukin-8 is a prognostic indicator in human hilar cholangiocarcinoma. Int J Clin Exp Pathol. 2015; 8(7):8376-84.

43 Ruys AT, Groot Koerkamp B, Wiggers JK, Klümpen HJ, ten Kate FJ, van Gulik TM. Prognostic biomarkers in patients with resected cholangiocarcinoma: a systematic review and meta-analysis. Ann Surg Oncol. 2014;21(2):487-500. 